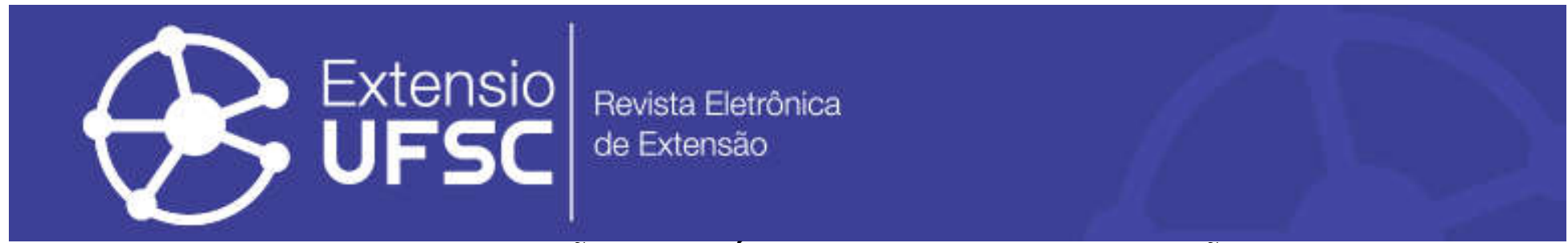

\title{
A PROMOÇÃO DE SAÚDE MENTAL EM IDOSOS NÃO- INSTITUCIONALIZADOS ATENDIDOS PELO SUS: GERAÇÕES DO FAZER SAÚDE
}

\author{
Marcello José Ferreira Silva \\ Universidade Federal do Pará \\ marcellofufpa@gmail.com \\ Nicole Morais Dillon \\ Universidade Federal do Pará \\ ninidillon@hotmail.com \\ Ana Clara Araújo \\ Universidade do Estado do Pará \\ anaacmaraujo@gmail.com
}

Bryan Skywalker Rufino Andrade Universidade Federal do Pará bryanrufino2@gmail.com

Luiz Wanderley Fontel dos Reis Junior Universidade Federal do Pará luiz.fontel@gmail.com

Luiz Lima Bonfim Neto Universidade Federal do Pará luizlbneto@gmail.com

Carla Mércia Souza Dacier Lobato Universidade Federal do Pará carlamercia@ig.com.br

\section{Resumo}

Agravos de saúde mental em pessoas idosas representam um problema de saúde pública mundial, à medida que aumentam a quantidade de hospitalizações e a taxa de mortalidade. O objetivo desse estudo foi de que, seguindo a linha de desenvolvimento de meios e processos de produção e de inovação, foram realizadas atividades de educaçãoem saúde mental em população idosa não-institucionalizada, usuárias de Unidades Municipais de Saúde na periferia de Belém (PA), entre março de 2017 e 2018. Percebeu-se interesse da população alvo em participar das discussões e das atividades. Assim, ficou evidente a relevância da utilização de estratégias de educação em saúde como forma de prevenção de doenças de saúde mental.

Palavras-chave: Idosos. Saúde Mental. Educação em Saúde. Prevenção.

\section{THE MENTAL HEALTH PROMOTIONWITH NON-INSTITUTIONALIZED ELDERLY ATTENDED BY SUS: GENERATIONS OF MAKING HEALTH}

\section{Abstract}

Mental health problems in the elderly represent a world wide public health problem as the number of hospitalizations and the mortality rate increase. The objective of this study was that, following the line of development of means and processes of production and innovation, educational activities in mental health were carried out in a non-institutionalized elderly population, users of Municipal Health Units. Target population to participate in discussions and activities. Thus, the relevance of using health education strategies as a way of preventing mental health diseases was evident.

Keywords: Seniors. Mental Health. Health Education. Prevention.

\section{LA PROMOCIÓN DE SALUD MENTAL CON PERSONAS MAYORES NO INSTITUCIONALIZADAS ATENDIDAS POR EL SUS: GENERACIONES PARA HACER SALUD}

\section{Resumen}

Los problemas de salud mental en los ancianos representan un problema de salud pública mundial a medida que aumenta el número de hospitalizaciones y la tasa de mortalidad. El objetivo de este estúdio fue que, siguiendo la línea de desarrollo de medios y procesos de producción e innovación, se llevaran a cabo actividades de educación en salud mental en una población de adultos mayores no institucionalizada, usuarios de Unidades Municipales de Salud. población objetivo para participar en debates y actividades. Por lo tanto, la relevancia de utilizar estrategias de educación para la salud como um medio para prevenir enfermedades de salud mental era evidente.

Palavras clave: Personas de Edad Avanzada. Salud Mental. Educación en Salud. Prevención. 


\section{INTRODUÇÃO}

Envelhecer é uma condição inerente à natureza humana, cujas mudanças ocorrem de maneira dinâmica nos âmbitos biológico, psicológico, social e cultural, em função do tempo. No decorrer dessas mudanças, são comuns agravos de saúde mental, os quais representam um grande problema de saúde pública mundial à medida que aumentam a quantidade de hospitalizações e a taxa de mortalidade (FALCÃO \& CARVALHO, 2018).

De acordo com Tavares et al. (2016), para assegurar que a população idosa apresente uma melhora na qualidade de vida, uma estratégia viável seria entender de que forma os processos degenerativos associados ao envelhecimento podem ser atenuados. Tendo isso em vista, é relevante compreender os fatores redutores de saúde de pacientes idosos na atenção primária à saúde. Nesse sentido, a depressão é um exemplo recorrente que apresenta impacto negativo expressivo na vida dos idosos (SANTOS et al, 2015).

Tal impacto, segundo Magalhães et al. (2016), pode ser observado em diversos aspectos da vivência da pessoa idosa, afetando desde a relação do idoso com sua família e equipe de saúde com a qual interage, até mesmo o desenvolvimento de atividades e os custos diretos e indiretos relacionados ao seu sustendo. Ademais, segundo Lima et al. (2016), a depressão se relaciona com numerosas outras comorbidades, interferindo tanto na adesão ao tratamento, quanto em processos inflamatórios e degenerativos muitas vezes relacionados a alterações cerebelares e cerebrais, além de ser, para Silva et al. (2017), considerada fator de risco para um pior prognóstico de doenças crônicas, a exemplo da diabetes e síndrome coronariana.

O conhecimento de tais fatos por profissionais de saúde se faz necessário, visto que há um elevado número de idosos depressivos que chegam aos serviços de saúde através da emergência, por vezes com outras queixas (LIMA et al, 2016). Outro ponto de importante destaque, emconformidade com Matos (2016), é a associação da depressão a alterações a níveis estruturais e funcionais do cérebro, as quais se expressam comprovadamente pelo decréscimo de zonas, como o hipocampo, cerebelo e córtex pré-frontal, originando,assim, mudanças no processamento de informações, percepção, aprendizagem, memória, atenção, vigilância, raciocínio e capacidade de solução de problemas, além de implicações no tempo de reação, tempo de movimento e velocidade de desempenho, produzindo, por conseguinte, intenso impacto no cotidiano, bem estar e, sobretudo, qualidade de vida da pessoa que envelhece.

No Brasil, com o crescente aumento da expectativa de vida, o processo de envelhecimento é gradativamente mais debatido e discutido cientificamente. Com isso, é notória a 
imprescindibilidade da ampliação de serviços, de pesquisas e de projetos direcionados ao atendimento das demandas de saúde mental da pessoa idosa (FALCÃO \& CARVALHO, 2018).

Nesse contexto, enfoca-se que as políticas públicas deverão ser efetivas e envolver setores distintos, como a saúde, a economia, o mercado de trabalho, a seguridade social e a educação. A perda da capacidade funcional é considerada um problema primordial para esses indivíduos, seja e natureza física ou mental imprescindíveis para a realização de atividades basilares e instrumentais de sua vida cotidiana. Logo, diante dessa importante modificação no perfil populacional do Brasil, expressa-se a necessidade de aperfeiçoar a capacitação de profissionais da atenção primária à saúde para identificação precoce de idosos vulneráveis à depressão.

Desse modo, o projeto objetiva discutir o tema da saúde mental em idososno contexto da atenção primária da saúde, local onde é possível entrar em contato direto com elementos culturais e sociais da comunidade. Por meio dessa ação de promoção de saúde, é possível estimular a consciência social sobre um tema pouco discutido e, ao mesmo tempo, relevante na saúde da população idosa. Por meio de um contato mais íntimo com essa população, é possível suscitar ações terapêuticas transformadoras.

\section{MATERIAS E MÉTODOS}

Trata-se de um relato de experiência desenvolvido entre os meses de março de 2017 e 2018, com idosos não-institucionalizados atendidos nas Unidades Municipais de Saúde (UMS) do distrito DAGUA: UMS do Jurunas, UMS da Cremação, UMS do Guamá, UMS da Terra Firme, do município de Belém (PA), e envolveu docentes e discentes dos cursos das áreas de Ciências da Saúde da Universidade Federal do Pará.

Além disso, os discentes envolvidos no projeto participaram de ciclos de Reuniões Científicas Trimestrais sobre a temática abordada. Esses encontros foram mediados pelo coordenador e pelos bolsistas do projeto, por meio da organização de seminários e de discussões sobre o tema, abertos a todos os discentes interessados e obrigatório para os envolvidos com o projeto. 


\section{RESULTADOS}

Sob a orientação dos profissionais envolvidos no projeto, foram elaboradas cartilhas educativas e panfletos de acordo com as demandas do público-alvo, com informações interativas sobre as temáticas trabalhadas. Além disso, foram solicitados e disponibilizados materiais impressos pela Secretaria Municipal de Saúde.

Nas UMS, as dinâmicas ocorreram em grupos e foi solicitado o apoio dos agentes comunitários de saúde para a divulgação das atividades propostas a fim de aumentar a adesão e, assim, foi possível dar início a grupos de idosos fixos em cada UMS. As atividades de educação em saúde foram feitas a partir de dinâmicas, respeitando as condições físicas e psicológicas de cada participante. Foi estimulado o compartilhamento de experiências, positivas e negativas para a construção coletiva do conhecimento em favor do combate da ocorrência de agravos de saúde mental. Foram utilizados vídeos educativos e distribuídos materiais gráficos para a multiplicação das informações aprendidas com amigos e com vizinhos dos idosos participantes.

Durante as ações, percebeu-se o interesse dos participantes a respeito de um assunto que não tinham tanto conhecimento. A metodologia de busca ativa dos participantes e a utilização de diferentes estratégias contribuíram para uma boa adesão dos idosos que participaram com questionamentos, relatos ou até mesmo apenas com a atenção.

Como mecanismo de avaliação continuada do projeto, foi solicitado dos discentes envolvidos a participação obrigatória nas reuniões científicas trimestrais, nas quais foram realizadas discussões sobre o progresso das atividades propostas, percepções e desafios enfrentados.

O nome do projeto - Gerações do Fazer Saúde - evoca a representação das diferentes gerações envolvidas nas atividades - executores e público-alvo -, unidos em favor da promoção de saúde, por meio da educação em saúde com a comunidade. Dessa forma, gerações se encontraram por meio da extensão, como mecanismo ativo de transformação social com um objetivo em comum: a saúde e bem-estar da pessoa idosa por meio do combate a agravos de saúde mental.

\section{DISCUSSÃO}

O aumento da expectativa de vida da população e a queda nas taxas de fecundidade e mortalidade resultaram no crescimento da proporção de idosos no Brasil. Segundo o Instituto 
Brasileiro de Geografia e Estatística (IBGE), o número de idosos deve passar de 14,9 milhões (7,4\% da população) em 2013 para 58,4 milhõesem 2060, correspondendo a 26,7\% da população (MAGALHÃES et al, 2016). O crescimento da população idosa provoca aumento de pessoas com risco de adquirir doenças neurológicas e psiquiátricas. Tais doenças constituem um grave de saúde pública e estão associadas a um piorana qualidade de vida (SANTOS et al, 2015).

Sendo assim, a Política Nacional de Saúde do Idoso recomenda a capacitação dos profissionais de saúde para o atendimento integral e assinala que essa deverá possibilitar a sistematização da atenção, com ênfase nas ações destinadas à promoção da saúde, à prevenção de incapacidades e à manutenção do desempenho cognitivo do adulto e idoso que vivem na comunidade (FALCÃO \& CARVALHO, 2018). Contudo, ainda não é garantida ao idoso uma participação ativa no seu processo saúde-doença e nem é garantia da saúde, o que dificulta a manutenção da saúde mental e a adesão aos tratamentos.

O envelhecimento populacional e a crescente demanda por serviços de saúde mental adequados exigem qualificaçãodos profissionais de saúde e compreensão sobre como enfrentar esses desafios. Nesse contexto, a Gerontologia dispõe de vários argumentos a favor da educação, da integração e da participação dos idosos na vida social, além de propor, estratégias relacionando a educação e o envelhecimento. Tais estratégias são consideradas importantes para a manutenção da funcionalidade, preservação e melhora do desempenho cognitivo e da qualidade de vida da pessoa idosa (LIMA et al, 2016).

Estudo realizado por Falcão \& Carvalho (2018) mostrou que a maioria dos idosos era do sexo feminino- e elas são mais propensas a prestar atenção nos sinais e sintomas e a procurar assistência à saúde quando comparadas com os homens. A maioria dos homens apresentou altas taxas de mortalidade relacionadas à violência, acidentes de trânsito e doenças crônicas.

Os idosos com incapacidade funcional também apresentaram maior prevalência de sintomas depressivos. O desenvolvimento de alguma limitação para realizar as atividades cotidianas pode gerar um sentimento negativo, o que pode levar a episódios depressivos. Da mesma forma, a redução do prazer nas atividades cotidianas, que é um dos sintomas da depressão, pode diminuir a capacidade de execução destas atividades, tornando-se extremamente difíceis (SANTOS et al, 2015).

Idosos com bons hábitos de saúde e engajados socialmente apresentam boa percepção de desempenho de memória e compreendem a importância de trabalhar o cognitivo (LIMA et al, 2016). A memória, por seu papel fundamental no processo de ensino-aprendizagem e na definição do indivíduo sobre sua identidade, constitui uma das funções cognitivas essenciais do ser humano. Sem ela, não seria possível, por exemplo, acumular e transmitir experiências a outras 
pessoas, o que promove a socialização da espécie humana. Há, portanto, necessidade de trabalhála e estimulá-la constantemente (TAVARES et al, 2016).

A autopercepção é a interpretação que uma pessoa faz do seu estado de saúde e das suas experiências no contexto de sua vida diária. Em geral, esse julgamento é baseado nas informações e nos conhecimentos disponíveis sobre saúde e doença, pode ainda ser mediado pela experiência prévia e pelo contexto social, cultural e histórico da pessoa. Este é um dos indicadores mais utilizados em estudos epidemiológicos e com idosos, sendo apontado como um bom indicador de bem-estar (SANTOS et al, 2015). Dessa forma, ações de promoção de saúde sobre a depressão é uma maneira de interferir na autopercepção do idoso a respeito da sua saúde mental, tornando-se uma ferramenta eficaz de prevenção da depressão.

A autoavaliação de saúde pode refletir um entendimento de mudanças biológicas e fisiológicas sutis que levam a perceber a própria saúde ou de forma mais positiva ou negativamente, podendo representar melhor o estado de saúde do que medidas objetivas (MATOS, 2016). E essas conclusões individuais apenas são possíveis a partir da transferência de conhecimento para o paciente e no estímulo deste, de maneira que o saber não seja algo inalcançável, e sim disponível para o público, de maneira interativa e simples. Isso demonstra a relevância de ação de saúde com um contato íntimo com a comunidade, para levar a uma melhor compreensão da sua saúde individual e, consequentemente, atuar na prevenção de agravos à saúde mental.

Ao se resgatar o papel da atenção primária como porta de entrada ao sistema de saúde, pautando-se pelo vínculo e pela longitudinalidade, pode-se depreender que ainda é preciso avançar frente ao cuidado à saúde do idoso, uma vez que, devido à cronicidade dos problemas, eles necessitam de acompanhamento sistemático. Ademais, é função comum de todos profissionais integrantes da atenção primária de saúde a promoção de atividades de conscientização para prevenção de doenças (BRASIL, 2017).

\section{CONSIDERAÇÕES FINAIS}

Dessa forma, é notória a importância de ações de educação em saúde mental, como uma forma de prevenção de depressão. Tendo em vista que oferecer ao idoso a autonomia em relação a sua saúde mental, por meio de informações precisas que foram passadas de maneira fácil e íntima, o indivíduo tem a oportunidade de compreender que possui papel principal em sua saúde 
mental e esta deve ser valorizada, tendo em vista que está relacionada com diversos outros fatores de saúde.

Sendo assim, este trabalho demostrou que a promoção de educação em saúde é uma ferramenta fundamental que está sendo pouco utilizada, porque edifica um conhecimento sólido de prevenção em saúde, pois fornece ao idoso o conhecimento que leva a uma mudança de comportamento e de autopercepção no meio de ação. Sendo assim, fazem-se necessárias medidas de ações de educação em saúde, para oferecer um cuidado com a saúde do idoso de maneira integral.

\section{REFERÊNCIAS}

ANDRADE, F. B.,et al. Promoção da saúde mental do idoso na atenção básica: as contribuições da terapia comunitária. Texto \& Contexto Enfermagem, Florianópolis, v. 19, n. 1, p. 129-136, 2010.

BRASIL. Portaria $\mathbf{n}^{\circ}$ 2.436, de 21 de setembro de 2017. Aprova a Política Nacional de Atenção Básica, estabelecendo a revisão de diretrizes para a organização da Atenção Básica, no âmbito do Sistema Único de Saúde (SUS). Brasília-DF: Ministério da Saúde, 2017. Disponível em: http://bvsms.saude.gov.br/bvs/saudelegis/gm/2017/prt2436_22_09_2017.html. Acesso em: 24 fev. 2019.

FALCÃO, D. V. S. \& Carvalho, I. S. Idosos, gênero e saúde mental. In: FALCÃO, D. V. S.; ARAÚJO, L. F. (orgs). Idosos e saúde mental. Campinas: Papirus Editora, 2018. Cap. 1, p.1123.

FERreirA, D. M. L., FALCÃO, D. V. S. A velhice e a Psicoterapia na Perspectiva de Pessoas Idosas In: FALCÃO, D.V. S., DIAS, C. M. S. B. Maturidade E Velhice Vol. i. Casa do Psicólogo, 2006. cap. 19, p. 423-443.

LIMA, A. M. P., RAMOS, J. L. S., BEZERRA, I. M. P., ROCHA, R. P. B., BATISTA, H. M. T., PINHEIRO, W. R. Depressão em idosos: uma revisão sistemática da literatura. Revista de Epidemiologia e Controle de Infecção, v. 6, n. 2, p. 96-103, 2016.

MAgalhães, J. M., CARvalho, A. D. M. B., CARVAlHO, S. M., ALENCAR, D. D. C., MOREIRA, W. C., PARENTE, A. D. C. M. Depressão em idosos na estratégia saúde da família: uma contribuição para a atenção primária. REME rev. min. enferm, v. 20, 2016.

MATOS, A. I. P. Efeito de dois programas: intervenção psicomotora e treino cognitivo, na Função Cognitiva e Depressão em Idosos. 2016. Dissertação de Mestrado. MS thesis. 2016.

SANTOS, C. A., RIBEIRO, A. Q., ROSA, C. D. O. B., RIBEIRO, R. D. C. L. Depressão, déficit cognitivo e fatores associados à desnutrição em idosos com câncer. Ciência \& Saúde Coletiva, v. 20, p. 751-760, 2015. 
SILVA, A. R., SGNAOLIN, V., NOGUEIRA, E. L., LOUREIRO, F., ENGROFF, P., GOMES, I. Doenças crônicas não transmissíveis e fatores sociodemográficos associados a sintomas de depressão em idosos. J BrasPsiquiatr, v. 66, n. 1, p. 45-51, 2017.

TAVARES, G. M. S., MULLER, D. V. K., FÃO, R. N., MANFREDINI, V., PICCOLI, J. D. C. E., SCNHEIDER, R. H. Análise da força de preensão palmar e ocorrência de quedas em idosas. Revista Brasileira de Ciência e Movimento, v. 24, n. 3, p. 19-25, 2016.

Recebido em: 05/05/2019

Aceito em: 16/07/2020 